\title{
Electromagnetic simulation of low-pass birdcage coil
}

\author{
Monika Mańko ${ }^{1, *}$ \\ ${ }^{1}$ Lublin University of Technology, Faculty of Electrical Engineering and Computer Science, Nadbystrzycka 38A, Lublin, Poland
}

\begin{abstract}
Magnetic resonance imaging (MRI) is a widely used non-invasive imaging modality that provides a variety of high-resolution soft-tissue contrast and functional information. The development of imaging technique employing the phenomenon of nuclear magnetic resonance is focused on obtaining the best possible tissue contrast, maximisation of ratio defined by signal-to-noise-ratio (SNR) and improving the image quality. SNR can be improved by the use of contrast agents and higher-field scanners, however these are better RF coils that often provide more significant gains. Using full-wave analysis and design tools becomes especially important when the coil dimensions approach a significant fraction of a wavelength. In this paper, modelling of RF coil is presented by using special-purpose software for electromagnetic simulations. The methodology of the research assumes making numerical calculations of RF coil. The presented approach of a design of radiofrequency (RF) coils for MRI scanners using lumped circuit modelling based techniques begins to fail at high frequencies, and therefore more accurate models based on the electromagnetic field calculations must be used. This paper presents simple simulations of the electric and magnetic field in LP birdcage coil.
\end{abstract}

\section{Introduction}

Radiofrequency (RF) magnetic field $\left(\mathrm{B}_{1}\right)$ homogeneity and signal-to-noise ratio (SNR) have always been important aspects of RF coil design in MRI. But the history of a technique of magnetic resonance started in 1946 when Bloch and Purcell $[1,2]$ proved that atomic nuclei with odd atomic and the mass number have nuclear magnetic moment different than zero. The interaction between nuclear magnetic resonance and external magnetic field with induction field $\mathrm{B}_{1}$ occurs due to the phenomenon of nuclear magnetic resonance at RF causing Zeeman nuclear effect -the splitting of energy levels [3]. In fact, detection of phenomenon of single nucleus resonance is impossible, only observation of resonance in some capacity connected with the whole sample is possible.

The research on the design of devices is aimed to improve the contrast of the resulting image. Most often, they are directed towards the construction of MR systems, which use increasingly higher values of the constant magnetic field. Physical phenomena cause that still a big challenge is the issue of choosing the right RF coil $[4,5]$, whose compatibility with the system determines the quality of imaging. Its design and construction are constantly improved to ensure the best possible sensitivity and the highest signal ratio. Birdcage coils (low-pass and high-pass) are widely used in magnetic resonance imaging applications due to their ability to operate in transmit/receive mode with high SNR and wide homogeneous fields [6-8]. Small animal imaging (which mostly uses birdcage resonators) is crucial to a majority of preclinical research [9-11]. This paper provides electromagnetic simulations of designed 8-legs LP birdcage coil for small animal imaging using MRI system.

\section{Methods}

\subsection{Radiofrequency Coil Design}

In this work, we aimed a simulation of the low-pass birdcage resonator tuned ultimately to the resonance frequency of ${ }^{1} \mathrm{H}$ nuclei at the $7 \mathrm{~T}$ constant magnetic field ( $\mathrm{f}_{\text {resonance }}=297 \mathrm{MHz}$, in this paper it was approximated to $300 \mathrm{MHz}$ ). The coil design in question is based on typical LP birdcage configuration with the number of legs $=8$.

The design and simulation of the birdcage were performed in the CST Microwave Studio software (CST AG, Darmstadt, Germany). The proposed coil design consists of three main components: coil (birdcage strips and former), filling phantom (imported from CST library) and shield. The coil geometrical parameters were chosen to fit the magnet core diameter, cover the whole body of a small animal (mouse) and have high filling factor: coil inner diameter $=36 \mathrm{~mm}$, leg length $=48.5 \mathrm{~mm}, \mathrm{~N}$ legs $=$ 8 , rung width $=3 \mathrm{~mm}$, end-ring segment width $=3 \mathrm{~mm}$. The capacitance values for 16 fixed-value capacitors which have to be placed in the gaps on the rungs were previously defined using the Birdcage Builder application: $\mathrm{C}=10.5 \mathrm{pF}$. These capacitance values were then introduced into the coil model in the CST.

RF shield tuning generally eliminates current imbalances and field distortions, improves $B_{1}$ field homogeneity and high-quality factors, as well as eliminates adjustable capacitors on the legs of the RF coil. RF shield tuning and inductive matching provide an isolated resonance structure which is both physically and

* Corresponding author: monika.manko@pollub.edu.pl 
electrically unattached [12-14]. The RF shield parameters of the proposed design are: RF shield inner diameter $=33$ $\mathrm{mm}, \mathrm{RF}$ shield diameter $=53 \mathrm{~mm}, \mathrm{RF}$ shield length $=120$ $\mathrm{mm}$.

The next step of coil design consisted in defining discrete ports. Red arrows in Fig. 1 represent discrete ports (16) used for the electromagnetic field simulations: impedance value $=50 \Omega$ (for S-parameter), voltage amplitude $=1 \mathrm{~V}$, and current value $=1 \mathrm{~A}$. Two stimulation ports were set on one end-ring and these ports were used to send the electric signals to the coil with the phase shift $=90^{\circ}$ in the EMC simulations.

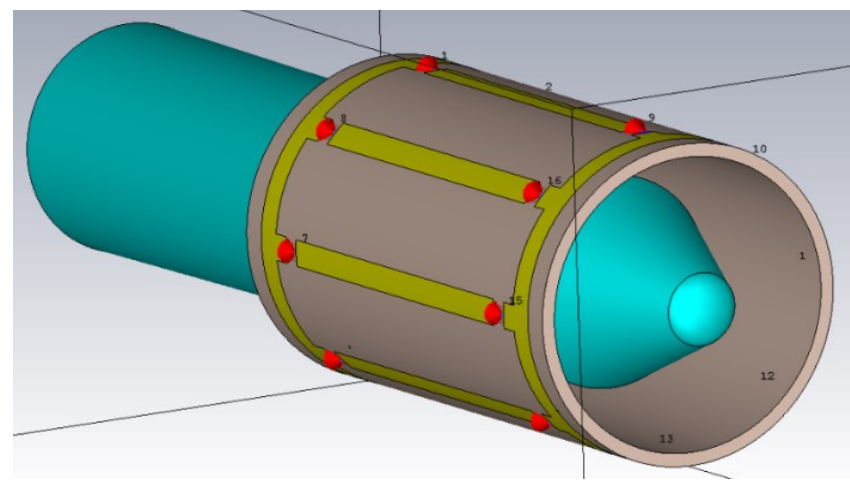

Fig. 1. View obtained from CST Studio illustrating the LP birdcage coil with the imported phantom.

The coil was tuned to $300 \mathrm{MHz}$ (for 7T MRI scanner approximately) and amounted to $50 \Omega$ (which is a characteristic impedance of coaxial cables used in MRI).

The results of the simulation included electric and magnetic fields generated by the coil, surface current distribution and also S-parameters.

\subsection{Electrical circuit model}

Having successfully designed the geometrical model of the RF coil including the application of appropriate materials (from the material library, in our work it is Copper, FR4-lossy, PEC, Vacuum and Muscle for the phantom), the next important part of the modelling was drawing a circuit model of the coil.

The electromagnetic simulations were performed using the Finite Integration Technique of CST Studio Suite. There were four types of capacitors used in our study: $\mathrm{Cr}, \mathrm{Ct}, \mathrm{Cm}, \mathrm{Cd}$. At the beginning of the drawing the circuit there were 16 identical capacitors $\mathrm{Cr}$ (the values was previously calculated in $10 \mathrm{pF}$ for each). Then it was necessary to add two external ports ( 1 and 2 in yellow rectangles in the schematic block) and replace two regular capacitors $(\mathrm{Cr})$ with matching capacitors $(\mathrm{Cm})$. For this purpose, we chose discrete ports number 1 and 3. Capacitors used in these simulations were modelled with additional resistances, which represented typical losses for lumped elements and losses inherent to soldering joints $(37,39)$. The final circuit designed in CST Studio is shown in the schematic view in Fig. 2. In the S-parameter method (Fig. 3), the network is assumed to have a linear response to small input signals. The meaning of the Sparameters is as follows:

-S11: the reflected power when a signal is applied at various amplitudes and frequencies to Port 1 ,

-S22: the reflected power when a signal is applied at various amplitudes and frequencies to Port 2 ,

-S12: the transmitted power recorded at Port 2 when a signal is applied to Port 1 ,

-S21: the transmitted power recorded at Port 1 when a signal is applied to Port 2 [15].

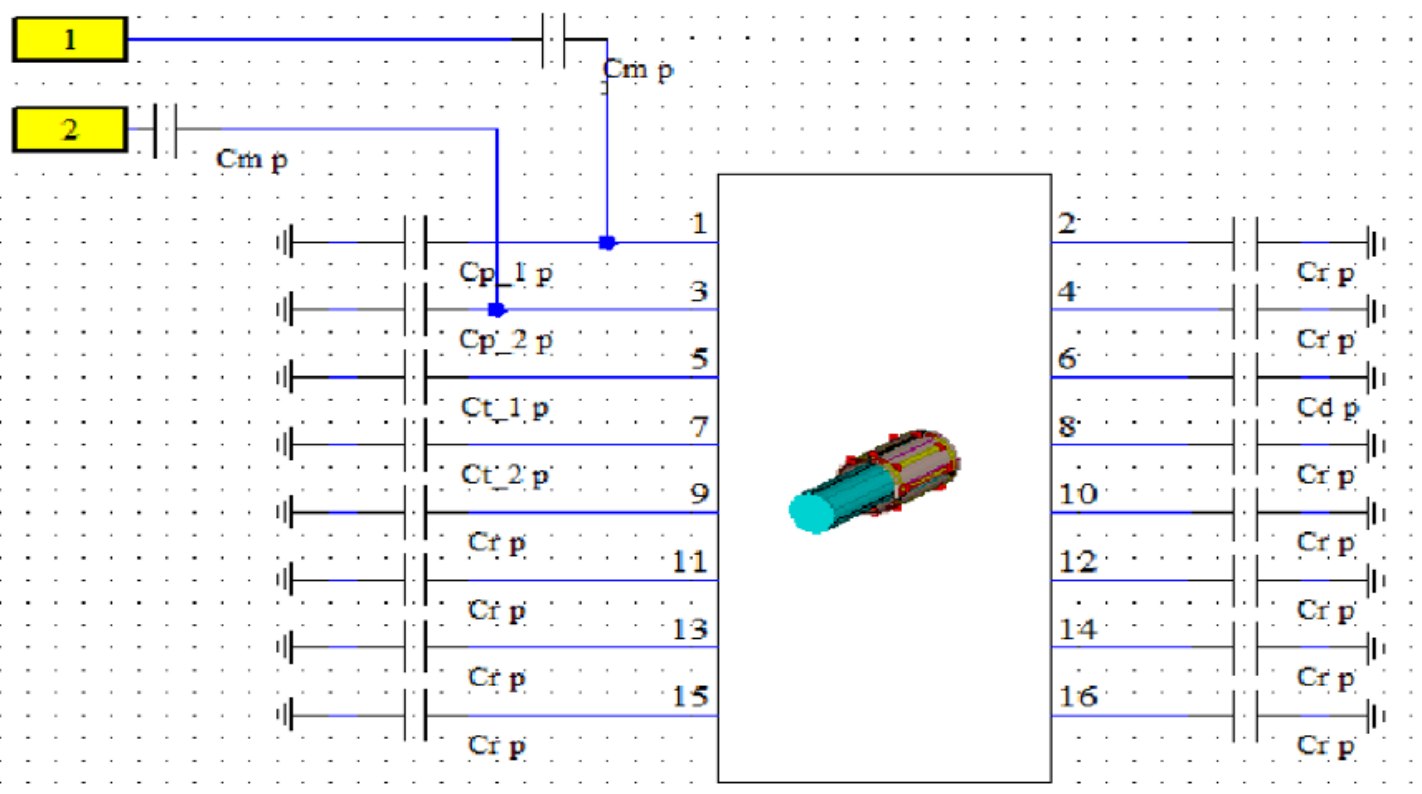

Fig. 2. Schematic circuit block drawing in CST Studio. 


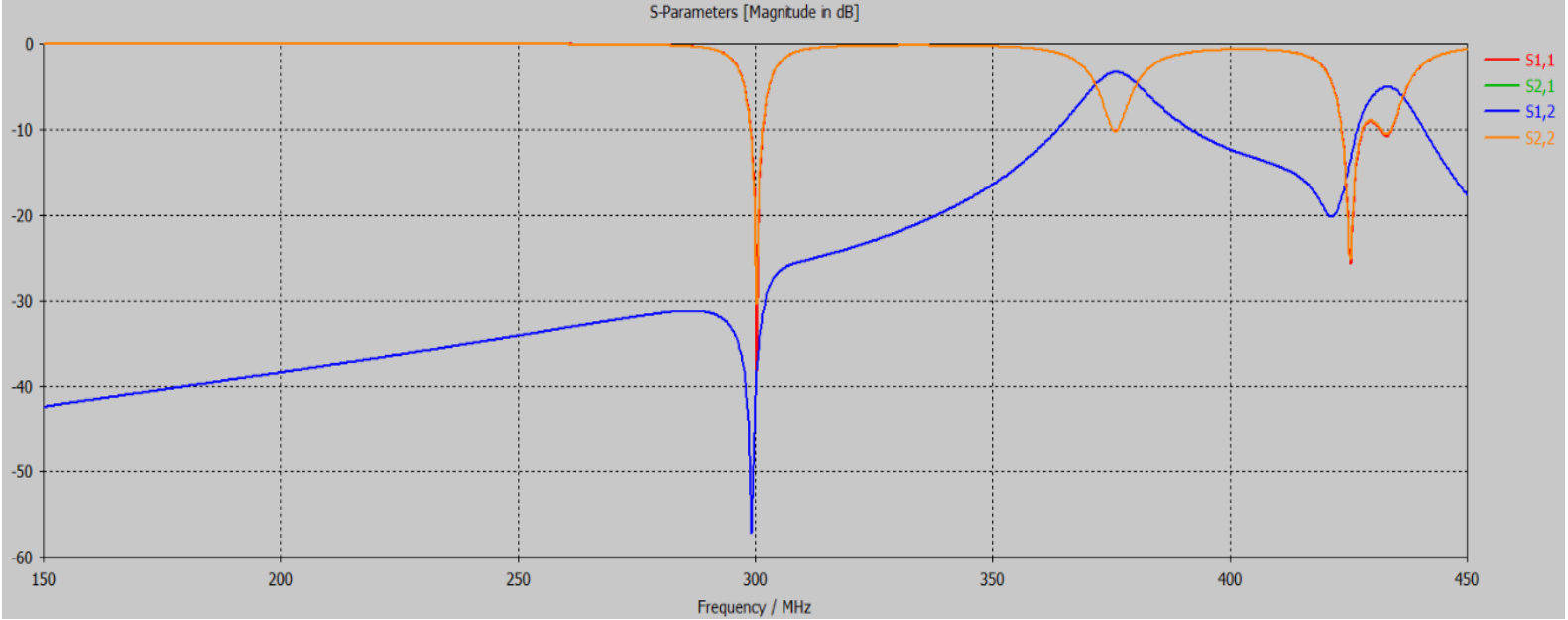

Fig. 3. S-parameters plot for the tuning LP birdcage coil in CST Studio.

Fig. 3 shows S-parameters plot for a tuned coil with good coupling at the desired frequency. In this case, S11 $=-4.84, \mathrm{~S} 21=-37.61, \mathrm{~S} 12=37.61$, and S22 $=-4.84$. In the CST Studio, each element of the coil circuit was tuned to the resonance frequency of $300 \mathrm{MHz}$ and matched to the impedance of $50 \Omega$. Failure to properly tune and match the coil may result in noisy images with poor contrast in the experiments $[16,17]$. In our study, we used a manual tuner for changing values of capacitors. CST environment has a tuner which can be open as an additional window. Tuning can refer to the values of variable parameters implemented in the circuit. The values of individual parameters have been chosen intuitively using sliders. Successful tuning is shown in Fig. 3 with the following values: $\mathrm{Cr}=10 \mathrm{pF}, \mathrm{Ct}=5.97 \mathrm{pF}, \mathrm{Cm}=4.51 \mathrm{pF}, \mathrm{Cd}=9.99 \mathrm{pF}$.

This setup was also used to calculate the electromagnetic field distribution described below.

\section{Results}

\subsection{Numerical EMF Simulations}

The RF field distribution depends on the details of the coil configuration. When the frequency increases, RF fields can interact more strongly with the sample, i.e. the animal tissues, rendering quasi-static approaches no more reliable for electromagnetic characterisation [18]. Numerical calculations described in this work can provide useful information to aid experimental low-pass birdcage coil design.

In most cases, it is very difficult to have access to the actual matching networks and to correctly reproduce them in the simulation; thus, it follows that the simulated Sparameter matrix can be quite different from the actual one.

In this study, the entire simulation works are made in the unloaded condition. But for the next studies, minimising the electric fields and the electromagnetic interaction around the probe will be crucial. The simple rules should be kept in mind during each stage of the probe design and construction:
- the inductance of the coil should be kept a low as possible in order to minimise the voltages,

- the length of wires should be kept short,

- the symmetric configuration should be used whenever possible [5].

Simulating an empty coil (as performed in this paper) will not provide a full picture of how it will work in practice. By placing an animal (or phantom) in the MRI scanner, it could load the coil and introduce sources of interference, which will affect the electromagnetic field distribution $[5,19]$.

A homogenous electric field around the birdcage coil at $300 \mathrm{MHz}$ is shown in Fig. 4. In addition, the real part of the electric flux is almost orthogonal to its imaginary part, which indicates the flux is rotating circularly.

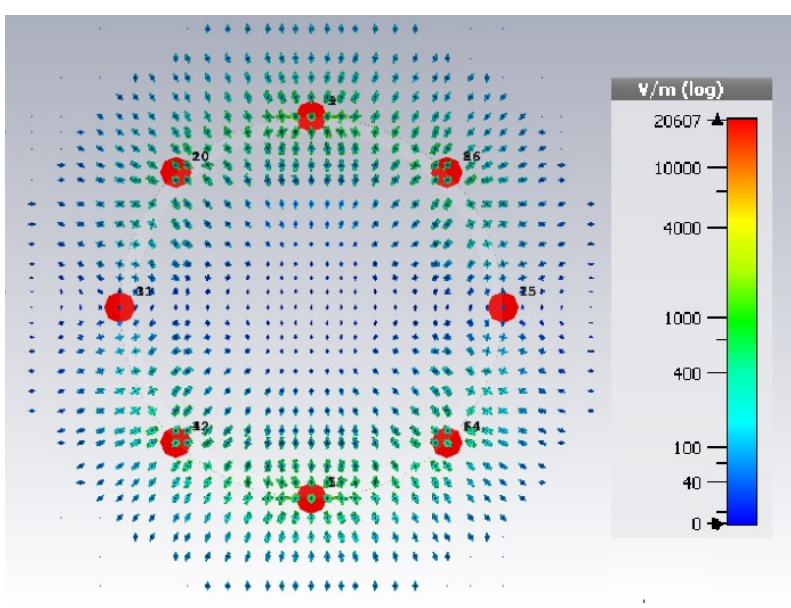

Fig. 4. Simulated electric field (E-field) vector plot.

Magnitude image of $\mathrm{H}$ at the central slice $(\mathrm{z}=0)$ for quadrature excitations is given in Fig. 5. The magnetic field distribution created by the RF birdcage coil is expected to reach the ideal homogeneity. Obviously, this depends on the number of leg or rung conductors.

Additionally, the simulated surface current plot (Fig. 6) and power loss density (Fig. 7) are also unloaded. 


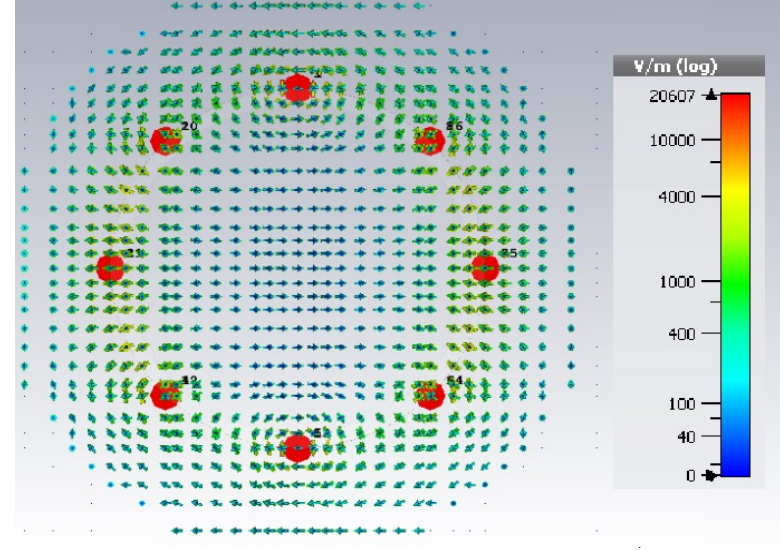

Fig. 5. Simulated magnetic field (H-field) vector plot.

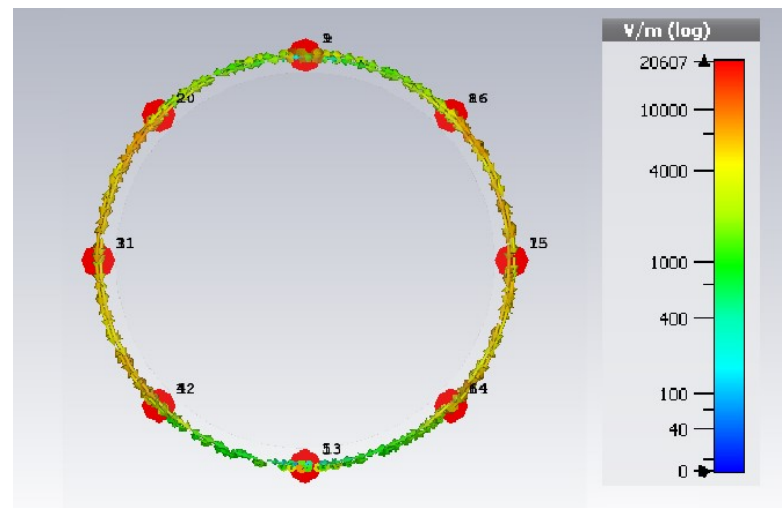

Fig. 6. Simulated surface current plot.

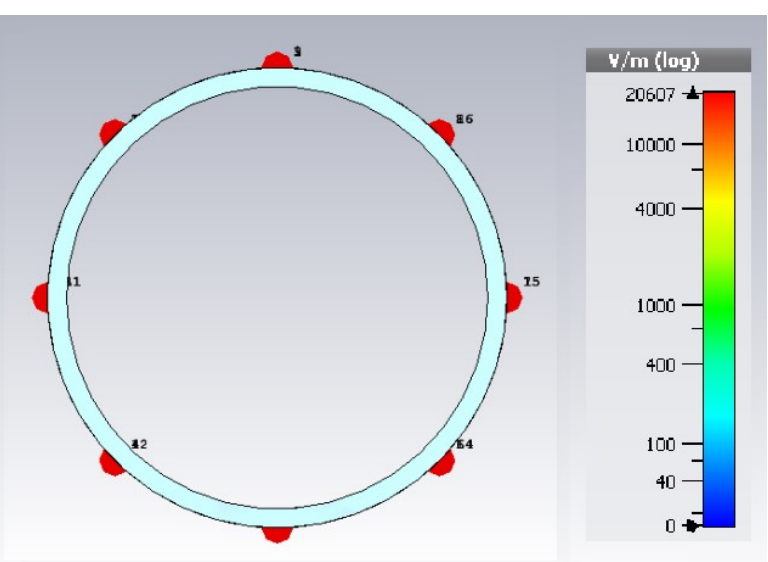

Fig. 7. Simulated power loss density plot.

\section{Summary}

A low pass birdcage coil designed to operate at $300 \mathrm{MHz}$ is simulated to show the electric field, magnetic field, surface current and power loss density. As described above, the coil is an 8-rung, low pass birdcage coil with a $36 \mathrm{~mm}$ inner diameter, $48.5 \mathrm{~mm}$ leg length, and a shield diameter of $53 \mathrm{~mm}$, shown in Figure 1.

The main purpose of numerical modelling is to assess the influence of specific RF coil model in the imaging diagnostics system, resonance modes, currents and distribution of magnetic field in resonance conditions, as well as identification of noise sources and dielectric losses.
To ensure that the coil produces a homogeneous field inside the subject, the optimiser would also be run with a model inside the coil, to find a usable field that takes into account the EM properties of the animal body. That would be the subject of our future studies [20].

The obtained results of distributions of electrical and magnetic field coupled with measuring circuit will serve to determine specific absorption rate (SAR) and signal to noise ratio (SNR) in the future research [21].

The simulation of RF birdcage coil allows (us) to optimise the process of coil development.

\section{References}

1. F. Bloch, W. Hansen, M. Packard, Phys. Rev. 69, 127 (1946)

2. E.M. Purcell, H.C. Torrey, R.V. Pound, Phys. Rev. 69, 37-38 (1946)

3. P.A. Bottomley P.A., Andrew E.R., Phys. Med. Biol. 23, 630-643 (1978)

4. W.A. Edelstein, J.M. Hutchison, G. Johnson et al., Phys. Med. Biol. 25 (1980)

5. J.T. Vaughan, J.R. Griffiths, RF Coils for MRI, (John Wiley \& Sons, 2012)

6. E.R. McVeigh, R.M. Henkelman, M.J. Bronskill, Med. Phys. 12, 586-591(1985)

7. S.B. Raval et al., NMR Biomed. 29(12), 1768-79 (2016)

8. M. Alecci, C.M. Collins, J. Wilson et al., Magn. Reson. Med. 49, 363-370 (2003)

9. D.E. Vincent, T. Wang, T.A.K. Magyar et al., Journal of Biomed. Eng. 11, 41 (2017)

10. Z. Chen, K. Solbach, D. Erni, A. Rennings, IEEE Trans. Microw. Theory Techn., 65 (2017)

11. M. Alecci, C.M. Collins, M.B. Smith, P. Jezzard, Magn. Reson. Med., 46(2), 379-85 (2001)

12. H. Wen, A. S. Chesnick, R. S. Balaban, Magn. Reson. Med., 32(4), 592-498 (1994)

13. B.A. Baertlein, O. Ozbay, T. Ibrahim et al., IEEE Trans. Biomed. Eng., 47, 535-546 (2000)

14. C.L. Chin, C.M. Collins, S. Li et al, Conc. Magn. Reson. 15, 156 (2002)

15. C.M. Collins, M.B. Smith, Magn. Reson. Med., 40, 847-856 (1998)

16. D. Wenz, A. Kuehne, T. Huelnhagen., et al., Magn. Reson. Med., 2018

17. F. David Doty, G. Entzminger, J. Kulkarni. et al., NMR Biomed., 20, 304-325 (2007)

18. K.N. Kim, S.D. Han, J.H. Seo, et al., Magn. Reson. Med. Science 16, 253-258 (2017)

19. J. Paska, J. Froehlich, D.O. Brunner. et al., Proc. Int. Soc. Magn. Reson. Med. 17, 30-38 (2009)

20. T. Santini, J. Kim, S. Wood, N. Krishnamurthy, et al., Magn. Reson. Imaging 45, 1-6 (2017)

21. B.K. Li, F. Liu, E. Weber, et al., NMR Biomed., 22(9), 937-951 (2008) 